This is the accepted manuscript of:

M. Cristani, F. Olivieri, and. Rotolo. 2017. Changes to Temporary Norms. In Proceedings of ICAIL'17, London, United Kingdom, June 12-16, 2017, pages 39-48.

DOI: $10.1145 / 3086512.3086517$

(c) ACM 2017. This version of the work is posted here for your personal use. Not for redistribution. The definitive Version of Record was published in https://doi.org/10.1145/3086512.3086517 


\section{Changes to Temporary Norms}

\author{
Matteo Cristani \\ Department of Computer Science, \\ University of Verona, Italy \\ matteo.cristani@univr.it
}

\author{
Francesco Olivieri \\ Data61, CSIRO, Australia \\ francesco.olivieriphd@gmail.com
}

\author{
Antonino Rotolo \\ CIRSFID, University of Bologna, Italy \\ antonino.rotolo@unibo.it
}

\begin{abstract}
Normative systems accommodate temporary norms of several types, which can also be modified in different, and codified ways. In this paper we address the problem of modifying temporary norms that are represented by means of the combination of two known formalisms in the current literature. The framework evolves from a known one, which provides a system of norms at two distinct layers, and represents changes at the two layers as means to provide room for the codified change types. This results in four novel operators that anticipate and extend norms in two different combined ways, by preserving or not the effects of the norms in the period of time generated by the temporal modifications. We study these new operators and show how they relate to the operators of annulment and abrogation analysed elsewhere.
\end{abstract}

\section{CCS CONCEPTS} -Applied computing $\rightarrow$ Law; Theory of computation $\rightarrow$
Logic;

\section{KEYWORDS}

Norm change modelling, Temporal Defeasible Logic, Representation of temporary norms

\section{INTRODUCTION}

The topic of norm change in the law has been extensively studied from theoretical viewpoint (starting from fundamental contributions such as Kelsen's [28] and Hart's [25], see also [31, 42])by mainly considering the structural and general aspects of legal dynamics-and from the formal standpoint (see, e.g., [1-4, 9, 10, $27,39,43]$ ) - where much attention has been paid on theory revision techniques which abstract from any temporal consideration: a rather comprehensive and recent overview on norm change and lawmaking can be found in [7]. Some interesting studies have been developed, too, in multi-agent systems (see [40]). However, despite some efforts to introduce temporal reasoning and parameters in the law (among the first attempts in the literature of AI\&Law, see, e.g., [30]), almost no effort has been devoted to explore-from a formal viewpoint-temporal aspects of explicit legal changes, i.e., those changes that are implemented through explicit modifications, namely, through norms which change other norms. To the best of our knowledge, the main contribution to this issue is still due only to Governatori and Rotolo (see, in particular, [21], which offers the fundamental machinery).

In many other fields the problems related to norm change have been dealt with, and in particular legal theorists pointed out many aspects of specific forms of norm dynamics [36, 38, 41] and dealt with problems related to the ways in which the norms change depending on their authoritative source and on the nature of change $[12,23,26]$.

Governatori and Rotolo [21] worked on the problem of changing norms by means of a formalism that is based on two layers: metarules are used to represent norm-making mechanisms that are allowed in a given legal system, and rules to represent norms by themselves. This approach significantly differs from those inspired by Alchourrón et al.'s work on AGM-like theory revision [2], which are focused on the dynamics of obligations and permissions.

Many other investigations [18, 19, 22] provided an analysis of the possible ways in which norms can change.

Although these investigations have been focusing on the main aspects of legal changes, which is still one underdeveloped point, they do not consider temporary norms, which have also been dealt with per se in the current literature of AI \& Law, but have not been studied as object of modifications.

Generally speaking, modifications can be either explicit or implicit. In the first case, the law introduces norms whose peculiar objective is to change the system by specifying what and how other existing norms should be modified. In the second case, the legal system is revised by introducing new norms which are not specifically meant to modify previous norms, but which change in fact the system because they are incompatible with such existing norms and prevail over them.

Some recent investigations have worked on problems related to ways in which changes in defeasible theories can be performed [15, $17,34]$. These studies, however, do not cover the explicit temporal knowledge aspect.

Norms are temporary when they have a pre-definite temporal extension, namely when they are issued with attached an expiration date, e.g. a date after which the legal provision will cease to be effective.

Norms treated by Governatori and Rotolo [21] do not possess temporal constraints regarding their expiration, whilst, in fact, many norms do have these kinds of properties. Norms with temporal constraints are named here temporary, where the name refers to 
the notion of temporal validity as the temporal interval in which a norm is applied, while the issuing time is the instant of the temporal axis in which the norm is issued. In this paper we make a very simple assumption on the relationship between the above mentioned temporal properties, which allows us to classify a wide variety of temporary norms, and consequently many forms of modifications.

Although the investigation which we base our work upon is rather complete in embedding the above defined modifications, there is one class of laws, and a corresponding class of modifications, that can only exist when we add explicit validity expiration time to the norms themselves. The major operations described in previous work [21] are annulment and abrogation. In this paper we prove that, by modifying the two layers in a slight way we can add a rather relevant expressive power to the formalism that, besides basic annulments and abrogations, accommodates temporal extensions and temporal anticipations, which both act on the temporal extension of a norm.

Governatori and Rotolo [21] dealt with norms that are issued at a given instant of time and persist onward. Facts of the world are derived in reality that can contrast with a norm at the instant in which they are derived. A norm is retroactive when time is mentioned explicitly in the norm itself in such a way that it covers derivability of facts in the past with respect to the issuing time. The derivation of facts that are consistent with a norm can be regarded as a form of compliance analysis, since the extension of the set of facts that are consistent is the complement of the violations. A norm has, therefore, a validity time, which is issued in the norm itself, and can be limited to some effects that are mentioned explicitly. When the effects of a norm are limited to an interval of time that is terminating in the future we say that the norm is temporary. Notice that when a norm acts in the past retroactively, we can assimilate this specific behaviour to regular norms with explicit time.

Within the limits of this investigation we can drive a new variant of the logic machinery for annulment and abrogation of norms [21], in order to understand the ways to codify changes to temporary norms. In fact, we have two distinct aspects of a norm in its issuing time: validity and effects. A norm is valid, in general, since its issuing time, whilst its effects can apply either in the past or in the future of that instant. Clearly, when we consider the moment in which a norm is suppressed, we can have different scenarios, thus giving rise to different suppression methods. First of all, in general, a norm can be annulled or abrogated. As referred to temporary norms, instead, we have:

- The norm terminates its validity interval;

- The norm is explicitly anticipated;

- The norm is explicitly extended;

- The norm is postponed.

When a norm is temporary, the two latter modifications are allowed, differently from the former operations that are possible for every type of norm, but with completely different effects from temporary to persistent norms.

Essentially, an annulment is a repeal, as it makes a norm invalid and removes it from the legal system. The norm is suppressed in a potentially retroactive way, namely it is not valid since a time preceding the time of the suppression (typically, since the very moment when the norm was enacted). This can happen for an explicit act of suppression, which can be either an act of the same issuing authority, or an act of a superior authority, or can be revoked, due to the violation of some principles of the law: the norm that is issued conflicts with other norms that are fundamental and unmodifiable. This can also occur because of a procedural nature, i.e. the norm issuing act is per se illegal or conflictual.

The modification called abrogation consists in suppressing only the effects, and it is usually not retroactive. However, abrogations are rather cumbersome with respect to temporary norms. Usually abrogations operate ex nunc and so do not cancel the effects that were obtained before the modification. If so, it seems that abrogations cannot operate retroactively besides specific cases. However, for temporary norms, an abrogation can be issued after the end of the temporal validity, and this can also be the case for annulment, though this case can be treated more easily. On the same time, there is a case of abrogation that is difficult to distinguish from annulment as it consists in suppressing the norm since its initial application. In this case, we have that the norm has no temporal validity but its effects can still be in act. This case, also known as leading to ghost norms, can occur as well for temporary norms.

There exist several examples of this kind of abrogations. For instance, we amy have in Italy a fiscal legal provision that is issued with validity on one year at the end of that year, but the tax payment is fixed in the further year. When this law is abrogated, its consequences in the future are prevented. However, if a temporary law is issued after the fiscal law with validity time in the past-a retroactive decree is often used to integrate fiscal laws in Italy-the validity time of that decree can be extended to the consequences of the fiscal law, which is therefore somehow abrogated in the past, but not in the future.

Furthermore, modifications of legal validity have similar effects of abrogations. For instance, when we anticipate the end of the validity of a law, we de facto abrogate it. Conversely, if two laws are in conflict, and the conflict resolution is delegated to a temporary law that suppresses the former law and issues the novel one, an extension of the validity of the former law can revive a conflict and thus generates an implicit abrogative effect. This is a common case of temporary laws.

Previous work [21] provided a detailed analysis of the reasons that lead to the definition of a model in which time is somehow abstracted away. However, this is not the case for systems that include temporary norms. Therefore we need to combine deadlines and the date-issuing approach developed in the above mentioned paper. We now present informal examples of the issues that can arise when we want to modify rules that have a temporary nature. Example 1.1 refers to a temporary norm that is issued with an explicit deadline that is modified further on, before its natural expiration date by anticipating the deadline, whilst Example 1.2 refers to a temporary norm that is modified by extending its expiration date.

Example 1.1. Every year, the national government in Italy issues norms to cover urgent matters. The Italian name for this legislative procedure is decretazione d'urgenza and the corresponding law is called decreto legislativo. When such a decree is issued, the Italian Constitution allows the law to hold for a maximum of sixty days. When the deadline is reached, the decree expires, unless the parliament has converted it into a regular national legislative act. 
Moreover, the parliament can cover it with a law that is issued with the explicit intention of overwhelming the decree. These laws are constantly opened with the act of anticipation for the deadline of the decree they are issued in substitution of. The decree is therefore suppressed in the date of issue of the new national law.

When this happens, usually this act of suppression cancels the norm and its effects onward without any form of survival for the effects of the norm itself. Further on we shall introduce a case in which the suppression preserves the consequences that are derived in the transitory phase. Clearly, government decrees are converted in national laws rather frequently. Very often the conversion occurs with changes, namely suppression of parts and substitution of others, and also obviously by introducing new parts. The conversion law also contains a suppression act for the decree that anticipates its expiration date. In Example 1.2 we analyse the inverse case.

Example 1.2. Regional governments in Italy have the power of publishing calls for projects that have been promoted by the European Union and distributed to the local authorities, in a fund dissemination operation that is driven by national regulations. A call for projects is a temporary norm, whose expiration date is fixed when issued. Rather often, however, these calls do not attract a sufficient number of admissible applications, and therefore, the calls are extended, by moving forward the expiration date. Very naturally, this extension preserves the consequences already in force at the moment of the extension date.

As Example 1.2 clearly shows, an act of temporal extension, which might look like a preservation act, is, in fact, an act of change that can be assimilated to an abrogation, or an annulment. Basically, there is in fact another transitory period (since the moment of the extension of the natural deadline) where the norms were not in force before the extension act, and should be enforced in one of the two possible ways. In the example, the law can be suppressed while allowing the subjects that have already applied to provide new documents, or simply extending the deadline by allowing other subjects to participate in the procedure.

In this paper, we address these issues using Defeasible Logic (DL) $[5,33]$. We shall provide, in Section 2, a brief overview of DL, we refer to the model developed by Governatori and Rotolo [21] and extend it with the notion of deadline to defeasible rules, similarly to the logical system studied by Governatori et al. [13]. Specifically, in Section 2 we briefly recall some aspects of defeasible logic, and then provide a sketch of temporal defeasible logic, and in Section 3 we discuss some properties of these logics. Further on we introduce a model of abrogation and annulment of norms that is also extended to cover the cases in which we modify the deadlines of temporary norms (temporal anticipation and temporal extension) in Section 5. Finally, Section 6 takes some conclusions and sketches future work.

\section{OVERVIEW OF DEFEASIBLE LOGIC}

$\mathrm{DL}$ is based on a logic programming-like language and it is a simple, efficient but flexible formalism dealing with many different intuitions of non-monotonic reasoning. DL is closely related to logic programming [6] and an argumentation semantics exists [14]. DL has a linear complexity [29] and also has several efficient implementations [8]. In addition, some preliminary works on legal modifications in DL have been proposed $[18,19]$ and further developed in the recent past $[16,21]$.

A defeasible theory $D$ is a structure $(F, R,>)$ where $F$ is a finite set of facts, $R$ a finite set of rules, and $>$ an acyclic superiority relation on $R$. Facts are represented as literals and are indisputable statements. A rule expresses a relationship between a set of premises and a conclusion. Facts, premises and conclusions of a theory are literals of a propositional logic, denoted by letters, prefixed or not with $\sim$ used to denote the negation. Rules can be strict, when they connect premises to conclusions in a classical way (arrow $\rightarrow$ ), defeasible rules (arrow $\Rightarrow$ ), when the conclusion is taken as plausible and defeaters (arrow $\leadsto$ ) when the conclusion is considered blocked by the premises.

The superiority relation $(>$ ) provides information about the relative strength of rules, i.e., about which rules can overrule which other rules. In this paper, we limit $>$ to act only on those rules that derive opposite conclusions.

Conclusions will be tagged by the defeasible proof tags $+\partial B$, meaning that we have a defeasible proof for $B$ and by $-\partial B$, meaning that it is not possible to give a defeasible proof for $B$. Analogously, $\pm \Delta B$ means that we have (we have not) a strict proof for $B$ (a proof is strict iff only facts and strict rules are used).

Straightforwardly, where $+\partial B$ holds, then $-\partial B$ does not, and $-\partial \sim B$ does.

A derivation is a finite sequence $P=(P(1), \ldots, P(n))$ of tagged literals satisfying four conditions, which correspond to inference rules for each of the four kinds of conclusion. $P(1 . . i)$ denotes the initial part of the sequence $P$ of length $i$.

Some notational conventions before presenting proof conditions for DL derivations. Each rule is identified by a unique label. $A(r)$ denotes the set of antecedents of a rule $r$, while $C(r)$ denotes its consequent. If $R$ is a set of rules, $R[B]$ denotes the set of rules in $R$ with consequent $B$. If $B$ is a literal, $\sim B$ denotes the complementary literal: if $B$ is a positive literal $C$ then $\sim B$ is $\neg C$; and if $B$ is $\neg C$, then $\sim B$ is $C$.

DL is a sceptical non-monotonic formalism: with a possible conflict between two conclusions, i.e., one is the negation of the other, DL refrains to take a decision and we deem both as not provable unless we have some more pieces of information that can be used to solve the conflict. Within the limits of our formalisation, the superiority relation is the only possible way to solve a conflict.

Defeasible proofs proceed in three phases: we first look for an argument supporting the conclusion we want to prove (an applicable rule for the conclusion). Second, we look for arguments for the opposite of what we want to prove. Third, we rebut all the counterarguments. This can be done by showing that the counterargument is not founded, i.e., some of the premises do not hold, or by defeating the counterargument, i.e., the counterargument is weaker than an argument for the conclusion we want to prove. We introduce below the proof conditions for defeasible derivability. All the formal definitions can be found in [5].

$+\partial$ : If $P(i+1)=+\partial B$ then either

(1) $+\Delta B \in P(1 . . i)$ or

(2.1) $\exists r \in R[B] \forall A \in A(r):+\partial A \in P(1 . . i)$ and

(2.2) $-\Delta \sim B \in P(1 . . i)$ and

(2.3) $\forall s \in R[\sim B]$ either 
(2.3.1) $\exists A \in A(s):-\partial A \in P(1 . . i)$ or

(2.3.2) $\exists t \in R[B]$ such that

$\forall A \in A(t):+\partial A \in P(1 . . i)$ and $t>s$.

\section{REVISING DEFEASIBLE THEORIES}

We briefly discuss in this section the problem of how to embed in DL some ideas from belief and base revision in order to capture annulment, abrogation, extension and anticipation. This has already been addressed, at first by Governatori and Rotolo [21, 35], and further on, with specific emphasis on superiority relation by Governatori et al. [16].

We assume that a defeasible theory represents the basic logical structure of a legal system $[18,19]$. In legal systems, indeed, norm conclusions can be obtained only if we do not have stronger norms attacking them [37], compliant with the notions expressed in the juridical principles of law superiority, as in lex superior, lex posterior, lex anterior and lex specialis.

All the above mentioned approaches tend to adhere to the principles of the so-called AGM postulates, as expressed in [2]. However, it has been widely discussed in the literature of non-monotonic systems that important issues arise that cannot be dropped off without negative consequences in the system itself. We here omit to describe the treatment of these aspects for the sake of space, and assume directly the notion of contraction and expansion of a DL.

Within the limits of our formalisation, the extension of a Defeasible Theory $T$ is defined as the pair $E(T)=\left(\partial^{+}(T), \partial^{-}(T)\right)$, where a literal $B$ is either $B \in \partial^{+}(T)$ or $\sim B \in\left(\partial^{-}(T)\right.$. Accordingly, when a literal is positively derived is $B \in \partial^{+}(T)$ and when it is negatively derived, $B \in \partial^{-}(T)$. A contraction of a Defeasible Theory $T$ is a new theory $T^{\prime}$, whose extension is included in the extension of $T$ whilst an expansion of $T$, on the contrary, is a theory whose extension includes the extension of $T$.

As widely investigated by Governatori and Rotolo [21], revision does not mimic how the law implements norm changes, since "new" rules are generated to reflect the changes. An alternative to revision based on belief sets is base revision [24, 32]. Base revision does not operate on the extension of a theory, but rather applies to the theory "generators", i.e., the non-logical axioms of the theory. This idea can be naturally coupled with partitioning the elements of a theory into "facts" and "rules", where the former cannot be revised, unless update is used, while the latter may be subject to revision. The fundamental idea of previous work on formalisms that accommodate norm change [21] is to capture changes in terms of base revision of a temporal defeasible logic, where temporal labels are added onto literals of a defeasible theory in order to capture two distinct types of rule temporalisation, namely transient and persistent. A transient rule holds on a given temporal instant of the timeline on which rules are issued whilst persistent rules hold since the instant of issuing.

In this paper we provide a limited extension to the above mentioned formalism by adding a notion of rule deadline as provided by Governatori et al. [13]. This is done by simply adding a new type of rules, temporary rules, which are issued at a given instant, and persist until a deadline. For the sake of space, we avoid to treat, in this paper, the complex and extended issues of the legal systems in their modification timeline, which was investigated in previous work [21]. Determining consequences of the introduction of the new rule type is matter of future work.

Governatori and Rotolo [21] suggest that the dynamics of a legal system $L S$ are more correctly captured by a time-series $L S\left(t_{1}\right)$, $L S\left(t_{2}\right), \ldots, L S\left(t_{j}\right)$ of its versions. Each version of $L S$ is called a norm repository $[18,19]$. In other terms we can look at these rule system as versions of the legal systems, in the same terms in which versions are introduced in database literature.

The passage from one repository to another is effected by legal modifications and by persistence [19], in the models presented so far in the literature. We introduce the possibility that a particular norm, whose nature is temporary, reaches its deadline, and therefore that the rule expires. Therefore, changes in legal systems can also occur because of modifications in the validity time of norms, or naturally because of the expiration of a temporary norm.

As already shown in previous investigations, dynamics of norm change and retroactivity need to introduce another time-line within each version of $L S$.

By the above model, we can directly construct different forms of modifications. When rules do not possess a time deadline, that is, they do not expire over the timeline of their validity, natural operations are annulment and abrogation. When, instead, a deadline is attached we have different conditions in the legal system.

One can argue that a simple approach to the solution of the problem of abrogation of temporary rules in a legal system can simply consist in the same operation of base revision that is performed for persistent rules. However, expiration has different effects than abrogation of persistent rules. The intent of someone who has provided a temporary rule is twofold. On one hand, she may have been issuing the law because of a special temporary need, for instance the situation of a natural disaster. In this case, the expiration of the norm has a dramatic effect, since persistence of its effects would be contrary to the will of the issuer. Therefore, we need to modify the way in which modifications are provided for persistent rules in order to accommodate reversal for temporary effects, only for the validity-time interval that lasts from the instant in which the abrogation takes place until the natural previous deadline of the norm, for those effects that would have been naturally extended on the temporal axis for persistent rules, and preserve, in the repository, the complete cancellation of the effects of the previously existent temporary rule since its expiration forever. This introduces two distinct ways of modifying the deadline of a temporary norm backward and two forward ones:

- Backward abrogation is the operation of deleting the effects of a temporary norm retroactively with respect to its natural expiration date. This can be viewed as a form of strong anticipation of the deadline itself, which has a mixed nature, for it is an abrogation of the future effects, but also of the previous ones, with respect to the deadline, therefore sharing with annulment the nature of retroactivity;

- Forward annulment is the operation of annulling the norm for its persistence in the instant in which the law is in force, and further on, but without suppressing the effects of the norm for its persistence in the past of the moment in which the act of suppression is issued. We can look at this as a weak anticipation. 
The general annulment operator consists in removing the rule since the instant of the suppression act for all the effects of the law, both in the past and in the future.

Symmetrically, we can have dual effects by the inverse operation of extension. If we extend a norm, we may have the following:

- Forward abrogation is the operation of deleting the effects of a temporary norm in the future of a new deadline, moved forward with respect to its natural expiration date. This can be viewed as a form of weak extension of the deadline itself, which has a mixed nature, for it is an annulment of the transitory effects, but not of the further ones, with respect to the deadline;

- Backward annulment is the operation of moving forward the norm expiration for its persistence without suppressing the effects of the norm for its persistence in the past of the moment in which the act of suppression is issued. We can look at this as a strong extension.

Again we have also abrogation, that consists in removing the rule since the instant of the suppression act for all the future effects of the law.

\section{A TWO-LAYERED FRAMEWORK FOR NORM ISSUING AND SUPPRESSION}

Previous work [21] presented some variants of temporal defeasible logic (TDL) [20]. In the proposed formalism, a literal in the logic has associated to it a timestamp $a^{t}$, meaning that $a$ holds at time $t$.

When we derive a conclusion this can be either persistent, namely such that when it holds at the starting and ending point of an interval then the conclusion itself holds in every instant of the interval itself. When such a property does not hold, then the conclusion is said to be transient.

Conversely, it is also possible to tag with temporal labels the rules themselves. For instance, $\left(r: a^{t_{a}} \rightarrow b^{t_{b}}\right)^{t_{r}}$ means that the rule $r$ is in force at time $t_{r}$, or in other words, we can use the rule to derive the conclusion at time $t_{r}$. The full semantics of this expression appeared in [21]. The language of TDL is based on a (numerable) set of atomic propositions Prop $=\{p, q, \ldots\}$, a set of rule labels $\left\{r_{1}, r_{2}, \ldots\right\}$, a discrete totally ordered set of instants of time $\mathcal{T}=\left\{t_{1}, t_{2}, \ldots\right\}$, the negation sign $\neg$, and the rule sign $\Rightarrow$ for defeasible rules and $\leadsto$ for defeaters. A plain literal is either an atomic proposition or the negation of it. Given a literal $l$ with $\sim l$ we denote the complement of $l$, that is, if $l$ is a positive literal $p$ then $\sim l=\neg p$, and if $l=\neg p$ then $\sim l=p$. If $l$ is a literal and $t$ is an instant of time, i.e., $t \in \mathcal{T}$, then $l^{t}$ is a temporalised literal. The natural interpretation of a temporalised literal $l^{t}$ is that $l$ is true at time $t$. If $l^{t}$ is a temporalised literal and $x \in[1, \infty)$, then $l^{(t, x)}$ is a duration literal. Again the natural interpretation of a duration literal $l^{(t, 1)}$ is that $l$ holds at time $t$, but nothing is assumed upon $l$ afterwards (and also before). Conversely, when $l^{(t, \infty)}$ is concluded, we assume that $l$ holds in $t$ and thereafter. Generally speaking, we say that $l^{(t, x)}$ is interpreted as $l$ holds since $t$ for $x$ instants of time. If $l^{(t, x)}$ is a duration literal, $y \in[1, \infty) t^{\prime} \in \mathcal{T}$, then $l^{(t, x)} @\left(t^{\prime}, y\right)$ is a fully temporalised literal, that, based on the durations $x$ and $y$ in the literals, is interpreted as $l$ is true at time $t$ for $x$ instants of time in the repository in force at time $t^{\prime}$ onwards for $y$ instants of time.
A rule is a relation between a set of premises, namely conditions of applicability of the rule, and a conclusion. Admissible conclusions are either literals or rules themselves building two classes of rules: meta-rules and proper rules. Meta-rules describe the inference mechanism of the institution on which norms are formalised, and fundamentally implement the issuing and suppression principles of the legal system.The intuition behind meta-rules is that meta-rules yield the conditions to modify a legal system. A temporalised rule is either an expression $(r: \perp)^{(t, x)}$ (the void rule) or $(r: \emptyset)^{(t, x)}$ (the empty rule) or $(r: A \Rightarrow B)^{(t, x)}$, where $r$ is a rule label, $A$ is a (possibly empty) set of temporalised literals, $\Rightarrow$ is a rule sign, $B$ is a duration literal, $t \in \mathcal{T}$ and $x \in[1, \infty)$.

On the above basis we extend duration literals to have also expiration time, by means of the expression $(r: A \Rightarrow B)^{(t, d)}$ with the meaning for the rule to start being valid on $t$ for $d$ instants after t. As a matter of fact, extended duration rules can incorporate basic duration rules as formalised in previous work [21]: we can assume 1 as the minimum duration, corresponding to transient durations, and a special symbol $\infty$ to represent indefinite durations.

Rather naturally, the only extended part of the system is the rule duration. We do not actually need to extend literals, since the representation of literals having transient symbolic duration is sufficient to capture any kind of needed literal at the layer used for timeline where the rules apply.

In fact we have two temporal dimensions for norms in a normative system. The first dimension is when the norm is in force in it, and the second is when the norm exists in the normative system. We avoid, in this paper, to introduce the full formalism, as it was discusses in previous work [21], and as should be developed henceforth. Basically, proper rules can be used to conclude fully temporalised literals, whilst meta-rules have proper rules as conclusions.

The basic formalism introduced here is an adaptation of Governatori and Rotolo [21]'s system. A legal system is represented by a temporalised defeasible theory, i.e. a structure $\left(\mathcal{T}, F, R^{\mathrm{nm}}, R^{\text {meta }}, R^{\mathrm{mod}}, \prec\right.$ ) where $\mathcal{T}$ is a totally ordered discrete set of time points, $F$ is a finite set of facts, i.e., fully temporalised literals, $R^{\mathrm{nm}}$ is a finite set of unmodifiable rules, $R^{\text {meta }}$ is a finite set of meta rules, $R^{\text {mod }}$ is a finite set of proper rules, and $<$, the superiority relation over rules is formally defined as $\mathcal{T} \mapsto(\mathcal{T} \mapsto$ Rules $\times$ Rules $)$.

An unmodifiable rule is a rule such that $\forall t, t^{\prime}, t^{\prime \prime}, t^{\prime \prime \prime} r^{t} @ t^{\prime}=$

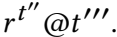

Basically, the derivation in TDL is a finite sequence of tagged expressions such that

(1) Each expression is either a temporalised rule or a temporalised literal

(2) Each tag is one of the following: $+\partial t @ t^{\prime},-\partial t @ t^{\prime}$;

(3) The proof conditions "defeasible rule provability" and "defeasible literal provability" given below are satisfied by the sequence $P$.

In particular:

\section{Defeasible Rule Provability}

If $P(n+1)=+\partial t_{d} @ t_{r} r^{t_{v}}$, then

1.1) $r_{v}^{\prime} @ t_{r}^{\prime} \in R^{\bmod }$ or

$\exists s^{t_{s}} \in R^{\text {meta }}\left[r^{t_{v}^{\prime}}\right]: \forall a^{t_{a}} \in A(s),+\partial t_{d}^{\prime} @ t_{r}^{\prime \prime} a^{t_{a}} \in P[1 . . n]$ and 
1.2) $\forall m^{t_{m}} \in R\left[\sim r^{t_{v}}\right]$ either

.1) $\exists b^{t_{b}} \in A(m):-\partial t_{d}^{\prime \prime} @ t_{r}^{\prime \prime \prime} b^{t_{b}} \in P[1 . . n]$ or

.2) $m^{t_{m}} \prec_{t_{d}}^{t_{r}} r^{t_{r}}$, if $r_{v}^{t_{v}^{\prime}} @ t_{r}^{\prime} \in R^{\mathrm{mod}}$ or $m^{t_{m}} \prec_{t_{d}}^{t_{r}^{d}} s^{t_{s}}$, if $r^{t_{v}^{\prime}} @ t_{r}^{\prime} \notin R^{\mathrm{mod}}$ or

.3) $\exists w^{t_{w}} \in R\left[r^{t_{v}^{\prime \prime}}\right]: \forall c^{t_{c}} \in A(w),+\partial t_{d}^{\prime \prime \prime} @ t_{r}^{\prime \prime \prime \prime} c^{t_{c}} \in P[1 . . n]$ and $m^{t_{m}} \prec_{t_{d}}^{t_{r}} w^{t_{w}}$

where

(1) if $r$ is persistent, then $t_{v}^{\prime} \leq t_{v}$; if $r$ is transient, then $t_{v}=t_{v}^{\prime}$;

(2) if $a^{t_{a}},\left(\right.$ resp. $\left.b^{t_{b}}, c^{t_{c}}\right)$ is persistent within the repository at $t_{r}$, then $t_{d}^{\prime} \leq t_{d}$ (resp. $\left.t_{d}^{\prime \prime} \leq t_{d}, t_{d}^{\prime \prime \prime} \leq t_{d}\right)$; if $a^{t_{a}}$ (resp. $b^{t_{b}}$, $\left.c^{t_{c}}\right)$ is transient within the repository at $t_{r}$, then $t_{d}^{\prime}=t_{d}$ ) (resp. $t_{d}^{\prime \prime}=t_{d}, t_{d}^{\prime \prime \prime}=t_{d}$ );

(3) if $a^{t_{a}}$ 's, $b^{t_{b}}$ 's and $c^{t_{c}}$ 's are persistent with respect to repositories (i.e., conclusions are persistent), then $t_{r}^{\prime \prime}, t_{r}^{\prime \prime \prime}, t_{r}^{\prime \prime \prime \prime} \leq$ $t_{r}$; otherwise $t_{r}^{\prime \prime}, t_{r}^{\prime \prime \prime}, t_{r}^{\prime \prime \prime \prime}=t_{r}$

(4) if $r_{v}^{t_{v}^{\prime}}$ and $s$ (i.e., facts, rules, and meta-rules) are persistent with respect to repositories, then $t_{r}^{\prime} \leq t_{r}$; otherwise $t_{r}^{\prime}=t_{r}$.

Temporary laws, namely, rules with definite duration, are treated as if they were persistent with respect to a number of repositories equal to the duration. Therefore we have that if $a^{t_{a}}\left(\operatorname{resp} . b^{t_{b}}, c^{t_{c}}\right)$ is persistent in the repository at $t_{r}$, and in any repository at $t$, with $t_{r}<t<t_{r}+d$, then $t_{d}^{\prime} \leq t_{d}$ (resp. $\left.t_{d}^{\prime \prime} \leq t_{d}, t_{d}^{\prime \prime \prime} \leq t_{d}\right)$.

\section{Defeasible Literal Provability}

The proof conditions for persistent and transient rules and literals in systems without temporary laws are fully provided in [21]. We report below the versions adopted for the same logical system. Below, $+\partial t_{d} @ t_{r} p^{t_{p}} \in P[1 . . n]$ represents only temporalised facts.

If $P(n+1)=+\partial t_{d} @ t_{r} p^{t_{p}}$, then

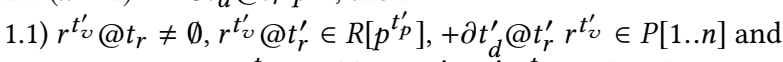
$\forall a^{t_{a}} \in A(r),+\partial t_{d}^{\prime} @ t_{r}^{\prime} a^{t_{a}} \in P[1 . . n]$, and

1.2) $\forall s^{t_{s}} \in R\left[\sim p^{t_{\sim} p}\right]$ if $+\partial t_{d}^{\prime \prime} @ t_{r}^{\prime \prime} s^{t_{s}^{\prime}} \in P[1 . . n]$, then either

.1) $\exists b^{t_{b}} \in A(s),-\partial t_{d}^{\prime \prime} @ t_{r}^{\prime \prime} b^{t_{b}} \in P[1 . . n]$ or

.2) $\exists w^{t_{w}} \in R\left[p^{t_{p}^{\prime \prime}}\right]$ such that $+\partial t_{d}^{\prime \prime} @ t_{r}^{\prime \prime} w^{t_{w}} \in P[1 . . n]$ and $\forall c^{t_{c}} \in A(w),+\partial t_{d}^{\prime \prime} @ t_{r}^{\prime \prime} c^{t_{c}} \in P[1 . . n]$ and $s^{t_{s}}<_{t_{d}}^{t_{r}} w^{t_{w}}$. where

(1) if $p$ is persistent, $t_{p}^{\prime} \leq t_{\sim p} \leq t_{p}$, otherwise $t_{p}^{\prime}=t_{\sim p}=t_{p}$;

(2) $t_{s}^{\prime} \leq t_{v}$, if $s$ is persistent, otherwise $t_{s}=t_{s}^{\prime}=t_{v}$;

(3) $t_{d} \leq t_{s}^{\prime}$, if $s$ is persistent, otherwise $t_{s}=t_{s}^{\prime}=t_{d}$;

(4) if conclusions are persistent over derivations (i.e., $+\partial t_{d}^{\prime} @ t_{r} p^{p_{t}}$ implies $+\partial t_{d} @ t_{r} p^{p_{t}}$ where $\left.t_{d}^{\prime}<t_{d}\right), t_{d}^{\prime} \leq t_{d}^{\prime \prime} \leq t_{d}$; otherwise $t_{d}^{\prime}=t_{d}^{\prime \prime}=t_{d}$;

(5) if conclusions are persistent over repositories, $t_{r}^{\prime} \leq t_{r}^{\prime \prime} \leq t_{r}$; otherwise $t_{r}^{\prime}=t_{r}^{\prime \prime}=t_{r}$.

We can distinguish two relevant properties: rule persistence and causal conclusion persistence. Usually, norms persist in a specific version of a legal system unless the norm is abrogated, annulled or expires.

We now provide examples of the different ways in which the abrogation and the annulment of temporary norms can affect the version of a legal system. Example 4.1 shows what happens when a temporary norm that is in force at a legal system is suppressed by an act of annulment.
Example 4.1. This is a fictional, but realistic example. Consider the case in which a government issues a temporary law that lasts, as happens in Italy, for sixty days. This temporary law suspends, for the period of validity, any obligation to pay the interests on the fines received in the year before. The procedure to obtain the privilege consists in making an online application and then waiting for a period of thirty days without a negative answer, based on a method that is called silenzio-assenso, namely silent-consent. During the validity period of the law several citizens apply for the privilege, but before the deadline of sixty days the decree is declared unconstitutional by the Constitutional Court, and therefore suppressed with an act of annulment. Obviously the annulment affects both those who applied and has received no negative answer being therefore able to use the privilege, which is however null, by the decision of the court, and, even more naturally those who applied but are still within the temporal horizon established in the decree.

The idea underlying the model of norm change developed in the literature [21], which we employ here and extend to cover the case of temporary norms, consists in a rule that can either be causal, namely such that it persists in further versions of a legal system, or not. The annulment of a norm simply consists in removing from the system the rule, or better, in transforming it into the empty rule. Furthermore, when a norm is abrogated, it is converted into a causal rule and then annulled. In this way, we obtain that the consequences of the rule persist over time in the future of the abrogation time, whilst the rule per se does not exist anymore.

Operations of temporal anticipation and temporal extension modify the expiration date. For the purposes of this paper we do not consider other modification operations that change the temporal extension of a temporary norms, and in one case, also persistent rules. This consists in changing the starting time of a rule, an operation usually named a postposition. For the purposes of this paper, as it will be clarified in the definition of the operations themselves, in Section 5, temporal anticipation and temporal extension are performed only before the expiration time of a temporary norm. Analogously, a postposition can be performed only before the starting time of a norm, independently of the rule being persistent or temporary.

\section{ANTICIPATIONS AND EXTENSIONS IN TDL}

In this section we recall the methods adopted in [21] to cancel norms in TDL by means of annulment and abrogation operators. Both modifications cancel norms from a legal system, but in completely different ways and mainly for completely different reasons and purposes. Let $L S\left(t^{\prime}\right)$ be the repository containing a modifiable rule $(r: A \Rightarrow B)^{\left(t_{v}, \infty\right)}$ such that $t^{\prime}<t_{v}<t^{\prime \prime}$, and $L S\left(t^{\prime \prime}\right)$ be the subsequent repository where we apply the modification of $r$, which is effective from a certain time $t_{a}$. Then $L S\left(t^{\prime \prime}\right)$ will contain $(r$ : $\emptyset)^{\left(t_{a}, \infty\right)}$. This makes the previous version of $r$ inapplicable in $L S\left(t^{\prime \prime}\right)$ from $t_{a}$, and so, there, we no longer obtain $B$ using $r:{ }^{1}$ condition (2.1) for Defeasible Literal Provability states that $(r: A \hookrightarrow B)$ is applicable if it is provable with that content, but this does not

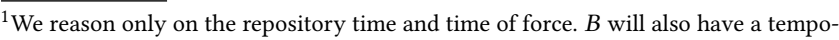
ral parameter and, if persistent, will hold from then onwards. We assume that $B$ is persistent and its time is slightly after $t_{a}$.
} 
hold after the modification (see condition (2.2) for Defeasible Rule Provability).

Although this seems to be a general solution to all problems arising with modification of norms forward, it does not deal with the basics of backward modifications. In particular, there is a fundamental difference between annulment and abrogation that sites upon the nature of the effect lock. In other terms conclusions of annulled rule are only derived in the repository in which the modification does not occur (see Figure 1(b)). This has been anticipated by Governatori et al. [19] and technically solved by the introduction of the operations of annulment and abrogation [21].

Figures 1(a) and 1(b) illustrate the approach to abrogation and annulment in TDL employed here. The solution proposed by Gover-

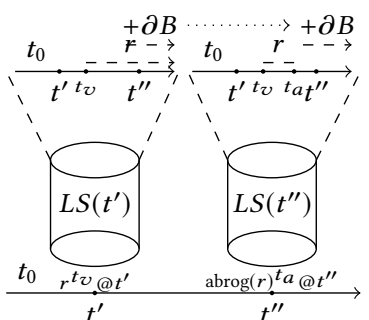

(a) Abrogation. In $L S\left(t^{\prime}\right)$ rule $r$ produces a persistent effect $B$. $B$ carries over by persistence to $L S\left(t^{\prime \prime}\right)$ even if $r$ is no longer in force.

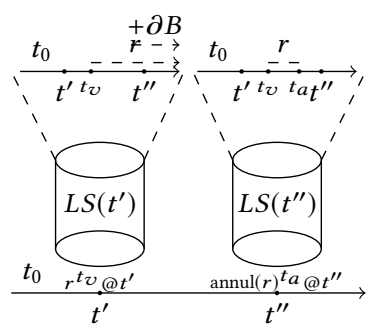

(b) Annulment. In $L S\left(t^{\prime}\right)$ rule $r$ is ap plied and produces a persistent effect $B$. Since $r$ is annulled in $L S\left(t^{\prime \prime}\right), B$ must be undone as well.
Figure 1: Abrogation and Annulment

natori and Rotolo [21] is as follows. Let the positive defeasible extension of a theory $T$ be the set $E^{+\partial}(T)=\left\{p^{t_{p}} @ t \mid T \vdash+\partial \# t^{\prime} @ t p^{t_{p}}\right\}$.

The abrogation of a rule $r$ at $t_{a}$ in repository $t^{\prime}$ is defined as follows:

$$
T_{r}^{a b r\left(t_{a}, t^{\prime}\right)}= \begin{cases}T & \text { if } r \notin E^{+\partial}(T) \\ \left(F, R^{\prime}, \prec\right) & \text { otherwise }\end{cases}
$$

where $R^{\prime}=R \cup\left\{\left(a b r_{r}: \Rightarrow(r: \perp)^{\left(t_{a}, \infty\right)}\right) @\left(t^{\prime}, \infty\right)\right\}$, where $t^{\prime}>t$. The abrogation simply terminates the applicability of the rule.

If we assume no persistency over repositories, the machinery that treats the annulment of persistent norms is rather simple, and consists in the following:

$$
T_{r}^{a n n-t r a n}\left(t_{a}, t^{\prime}\right)= \begin{cases}T & \text { if } r \notin E^{+\partial}(T) \\ \left(F, R^{\prime}, \prec\right) & \text { otherwise }\end{cases}
$$

where $R^{\prime}=R \cup\left\{\left(m r: \Rightarrow(r: \emptyset)^{\left(t_{a}, \infty\right)}\right) @\left(t^{\prime}, \infty\right)\right\}$ Thus to annul

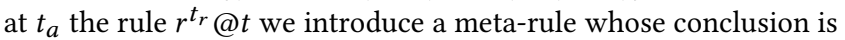
the empty rule. Though apparently tricky this approach eliminates the rule from the system, but simultaneously solves the problem of persistency of the effects over time, which should be prevented. The above, however, does not apply directly when the persistency over repositories is permitted. In particular, given a set of duration literals $D$, a set of temporalised literals $T$ and a total discrete ordered $(\mathcal{T},<)$, we define

$$
\begin{aligned}
D \cap_{(\mathcal{T},<)} T= & \left\{l^{t} \in T \mid \exists l^{\left(t^{\prime}, x\right)} \in D: t^{\prime}=t \text { if } x=1,\right. \\
& \text { and } \left.t \leq t^{\prime} \text { otherwise }\right\}
\end{aligned}
$$

For the sake of simplicity in the definition below the rules are the conclusion of a meta-rule (each with a unique name), thus the expression $\left(r: a_{1}, \ldots, a_{n} \Rightarrow b^{t_{b}}\right)^{t_{r}} @ t$ represents the abbreviation of the meta-rule (with empty body) ( $m r: \Rightarrow\left(r: a_{1}, \ldots, a_{n} \Rightarrow\right.$ $\left.b^{t_{b}}\right)^{t_{r}}$ @ . For a literal $l$, ann $(l)$ is a new literal not occurring in that theory.

Given a duration literal $b^{(t, x)}$ (where $\mathrm{x}$ is either 0 or $\infty$, and a theory $T$, we define the dependence set, i.e., the set of literals (called critical literals) potentially depending on it, as follows:

$$
\begin{array}{r}
\operatorname{Dep}\left(b^{(t, x)}\right)=\left\{b^{(t, x)}\right\} \cup\left\{c^{\left(t_{c}, x_{c}\right)} \mid \exists r \in R: C(s)=c^{\left(t_{c}, x_{c}\right)} \wedge\right. \\
\left.A(r) \cap_{(\mathcal{T},<)} \operatorname{Dep}\left(b^{(t, x)}\right) \neq \emptyset\right\}
\end{array}
$$

Then, if the annulment applies at $t_{a}$ in repository $t^{\prime}$

$$
T_{\left(r: a_{1}, \ldots, a_{n} \Rightarrow b^{\left(t_{b}, x\right)}\right)^{t_{r} @ t}}^{a \operatorname{annul}\left(t_{a}, t^{\prime}\right)}= \begin{cases}T & \text { if } r \notin E^{+\partial}(T) \\ \left(F, R^{\prime}, \prec^{\prime}\right) & \text { otherwise }\end{cases}
$$

where

$$
\begin{gathered}
R^{\prime}=R \cup\left\{(r: \emptyset)^{\left(t_{a}, \infty\right)} @\left(t^{\prime}, \infty\right),\right. \\
\left(r^{\sim}: \Rightarrow \sim b^{\left(t_{b}, x\right)}\right)^{\left(t_{a}, \infty\right)} @\left(t^{\prime}, 1\right), \\
\left.\left(r^{a n n}: \Rightarrow \operatorname{ann}(b)^{\left(t_{b}, x\right)}\right)^{\left(t_{a}, \infty\right)} @\left(t^{\prime}, 1\right)\right\} \\
\cup\left\{\left(s^{r e p}: A(s)-\operatorname{Dep}(C(r)) \cup\right.\right. \\
\left\{\operatorname{ann}(a)^{t_{a}} \mid a \in A(s) \cap(\mathcal{T},<) \operatorname{Dep}(C(r))\right\} \Rightarrow \\
\left.\operatorname{ann}(C(s))^{\left(t_{a}, \infty\right)}\right)^{\left(t_{a}, 1\right)} @\left(t^{\prime}, 1\right), \\
\left(s^{a n n}: \operatorname{ann}(C(s))^{t_{a}} \Rightarrow \sim C(r)\right)^{\left(t_{a}, \infty\right)} @\left(t^{\prime}, 1\right) \mid \\
\text { if } A(s) \cap(\mathcal{T},<) \operatorname{Dep}(C(r)) \neq \emptyset\} \\
\cup\left\{\left(s^{n a n}: A(s) \Rightarrow \sim a n n(C(r))^{\left(t_{a}, 1\right)}\right)^{\left(t_{a}, 1\right)} @\left(t^{\prime}, 1\right) \mid\right. \\
\text { if } C(s) \in \operatorname{Dep}(C(r)) \wedge A(r) \cap(\mathcal{T},<) \operatorname{Dep}(C(r))=\emptyset\} \\
<^{\prime}=<\cup\left\{\left(t^{\prime}, t_{a}, r^{r e p}, r\right) \mid r \in R\right\} \cup\left\{\left(t^{\prime}, t_{a}, r^{n a n}, s^{a n n}\right) \mid r, s \in R\right\}
\end{gathered}
$$

The idea is to replace a rule with a a new one that has been obtained as follows:

- For every rule where literals depending on the conclusion of the rule to be annulled occur in the antecedent, we create a copy of the rule where all critical literals are replaced by auxiliary literals.

- Moreover, for each critical literal its auxiliary literal is the body of a defeater for the complement of the critical literal.

- Finally, for each rule for a critical literal different from the conclusion of the rule to be annulled where no critical literal appears in the antecedent, we create a defeasible rule with the same body and as conclusion the complement of the critical literal.

The operations of abrogations and annulments apply to temporary laws in the same way in which they apply to persistent rules, but on the basis of the generation of auxiliary objects that are temporary rules. In particular, abrogation operations are defined as follows.

\section{Forward abrogation}

$$
\left.T\left[d^{\prime}\right]\right]_{\left(r: a_{1}, \ldots, a_{n} \Rightarrow b^{\left(t_{b}, d\right)}\right)^{t_{r}} @ t}^{f a b r\left(t_{a}, t^{\prime}\right.}= \begin{cases}T & \text { if } r \notin E^{+\partial}(T) \\ \left(F, R^{\prime},<^{\prime}\right) & \text { otherwise }\end{cases}
$$

- We generate two new temporary rules, $r^{\prime}$ and $r^{\prime \prime} . r^{\prime}$ is the extension of $r$ (obtained by applying the extension operator), and $r^{\prime \prime}$ is the same rule but starting in the deadline time of $r$ and ending in the deadline time of $r^{\prime}$. Further 
on, we abrogate $r^{\prime \prime}$, and build a superiority relation between the empty rule used to substitute $r^{\prime \prime}$ and $r^{\prime}$ in the repositories between the deadline of $r$ and the deadline of $r^{\prime}$.

In order to illustrate how the introduced method works, we formalise here the forward abrogation.

Example 5.1. Consider a law that is issued on the first of December 2015, and supposed to hold since January 2016 until the end of June 2016, which introduces a fishing licence for ponds. The law allows citizens to fish without licence until the deadline, provided that they obtain a licence by that date, otherwise they will get fined. By the end of May 2016, due to inefficiency in licence issuing, the government issues an extension that postpones the effects of the law, thus allowing the citizens to fish without a licence until July 2016, but without changing the deadline for application for licences. We represent the law original issue in the formalism as $r^{(1 / 1 / 2016,6 \text { months })} @(1 / 12 / 2015,7$ months $)$. A forward abrogation consists of the following steps:

- Generate, by the extension operator, a new rule

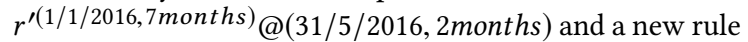

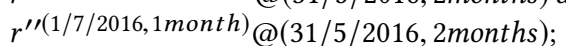

- We abrogate $r^{\prime \prime}$ and substitute it with the empty rule $\left(r^{\prime \prime \prime}\right.$ : $\emptyset)^{(1 / 7 / 2016,1 \text { month })}$;

- We establish $\left(r^{\prime \prime}>r^{\prime}\right) @(1 / 6 / 2016,1$ mont $h)$.

\section{Backward abrogation}

$$
T\left[d^{\prime}\right]_{\left(r: a_{1}, \ldots, a_{n} \Rightarrow b^{\left(t_{b}, d\right)}\right)^{t_{r}} @ t}^{b a b r\left(t_{a}, t^{\prime}\right)}= \begin{cases}T & \text { if } r \notin E^{+\partial}(T) \\ \left(F, R^{\prime}, \prec^{\prime}\right) & \text { otherwise }\end{cases}
$$

- We generate two new temporary rules, $r^{\prime}$ and $r^{\prime \prime} . r^{\prime}$ is the anticipation of $r$, obtained by applying the anticipation operator, and $r^{\prime \prime}$ is the same rule but starting in the deadline time of $r$ and ending in the deadline time of $r^{\prime}$. Further on, we abrogate $r^{\prime \prime}$, and build a superiority relation between the empty rule used to substitute $r^{\prime \prime}$ and $r^{\prime}$ in the repositories between the deadline of $r^{\prime}$ and the deadline of $r$.

Moreover, anticipations and extensions can occur only for rules that are in force at the moment of the operation, and therefore, are done within the same repository. The basic idea of an anticipation/extension is that a rule is operated on the one hand by

$$
T\left[d^{\prime}\right]_{\left(r: a_{1}, \ldots, a_{n} \Rightarrow b^{\left(t_{b}, d\right)}\right)^{t_{r}} @ t}^{\text {anticipate }\left(t_{a}, t^{\prime}\right)}
$$

that changes the duration $d$ to $\left(d+d^{\prime}\right)$, whilst, on the other hand,

$$
\left[T\left[d^{\prime}\right]_{\left(r: a_{1}, \ldots, a_{n} \Rightarrow b^{\left(t_{b}, d\right)}\right)^{t_{r}} @ t}^{\operatorname{extend}\left(t_{a}, t^{\prime}\right.}\right.
$$

that changes the duration $d$ to $\left(d-d^{\prime}\right)$. Without specification of what to do with anticipated effects (when extending) and with eliminated future effects (when anticipating) we can assume that these operators act in a given way, chosen as default; we prefer to provide distinct definitions for the combinatorial four models. The definition of the methods used to perform the operation are given out in an informal way since the notation is cumbersome. Moreover these correspond to operations on the basic rules generated by the abrogation and annulment rules. Regarding annulment, the structure is almost identical to the extensions provided by the abrogation operations.

\section{Forward annulment}

$$
\left.T\left[d^{\prime}\right]\right]_{\left(r: a_{1}, \ldots, a_{n} \Rightarrow b^{\left(t_{b}, d\right)}\right)^{t_{r}} @ t}^{\text {fannul }\left(t_{a}, t^{\prime}\right.}= \begin{cases}T & \text { if } r \notin E^{+\partial}(T) \\ \left(F, R^{\prime},<^{\prime}\right) & \text { otherwise }\end{cases}
$$

- We generate two new temporary rules, $r^{\prime}$ and $r^{\prime \prime}$. $r^{\prime}$ is the extension of $r$, obtained by applying the extension operator, and $r^{\prime \prime}$ is the same rule but starting in the deadline time of $r$ and ending in the deadline time of $r^{\prime}$. Further on, we annul $r^{\prime \prime}$, and build a superiority relation between the empty rule used to substitute $r^{\prime \prime}$ and $r^{\prime}$ in the repositories between the deadline of $r$ and the deadline of $r^{\prime}$.

\section{Backward annulment}

$$
T\left[d^{\prime}\right]_{\left(r: a_{1}, \ldots, a_{n} \Rightarrow b^{\left(t_{b}, d\right)}\right)^{t_{r} @ t}}^{\operatorname{bannul}\left(t_{a}, t^{\prime}\right)}= \begin{cases}T & \text { if } r \notin E^{+\partial}(T) \\ \left(F, R^{\prime}, \prec^{\prime}\right) & \text { otherwise }\end{cases}
$$

- We generate two new temporary rules, $r^{\prime}$ and $r^{\prime \prime}$. $r^{\prime}$ is the anticipation of $r$ obtained by applying the anticipation operator), and $r^{\prime \prime}$ is the same rule but starting in the deadline time of $r^{\prime}$ and ending in the deadline time of $r$. Further on, we annul $r^{\prime \prime}$, and build a superiority relation between the empty rule used to substitute $r^{\prime \prime}$ and $r^{\prime}$ in the repositories between the deadline of $r^{\prime}$ and the deadline of $r$.

To cover the cases with examples, Example 1.1 is a backward abrogation, whilst Example 1.2 is a forward annulment. Examples 5.2 and Example 5.3 cover the other two cases.

Example 5.2. A government decree is issued that allows companies to subtract a fixed amount to their monthly tax payments for three months, when the company has declared the need to do so in the first month of the decree. After two months the decree deadline is postponed of three further months. Clearly, the extension allows the companies that have presented the declaration of necessity state to use the norm, and does not allow further requests, as the previously established date has already expired.

Example 5.3. In recruiting acts of the American Government, it is established a term for applications for a position. The expiration date of applications can be anticipated only if the position is filled in advance. In this case, the anticipation is hard. for it prevents to derive conclusions from the moment of issues onward.

We can now prove a few results regarding the ways in which we can map anticipations and extensions in the basic norm change setting of TDL. The first result shows that temporary rules can be accommodated into the system presented in [21]. In particular, in the claim of the theorems below, we name direct consequence of a rule the set of all literals that cannot be derived in the repository in which the rule is applied without assuming those literals or a set of other literals and the rule itself.

To accommodate temporary norms in our system we can simply abrogate them at the expiration date. A system like the one we present in this paper is named TDL-T, for it is a TDL with temporary norms, whilst a system with temporal features along with defeasible machinery is named a TDL system. 
THEOREM 5.4. Every TDL-T system $T$ can be translated deterministically into a TDL system $T^{\prime}$ in linear time on the number of literals.

The proof of the claim of Theorem 5.4 consists in showing that a temporary rule can be substituted by the rule itself made persistent on the repository corresponding to the issue, and, if the system has this property, it is persistent over repositories (or an abrogation operation executed at the expiration date, i.e, that takes place at the expiration date, but is programmed synchronously with the norm issuing time). The number of repositories involved in this process amount at a maximum of elements that can be the number of admissible changes. The admissible changes involve at most once, all the literals. Therefore the translation is linear in the number of literals.

Moreover, we need to know whether the revision process that is performed against persistent rules can be also applied to combinations of temporary rules. We are now ready to claim the two projection results for temporary rules.

THEOREM 5.5. Forward annulment and backward abrogation of a temporary rule in a TDL-T system $T$ are meta-rule revision for the corresponding TDL system.

THEOREM 5.6. Forward abrogation and backward annulment of a temporary rule in a TDL-T system $T$ are rule revision for the corresponding TDL system.

More precisely, we can provide these basic operations in a TDL system, namely the contraction and expansion of a rule (or a metarule), only for those rules and meta-rules that have been generated to provide the mapping from a TDL-T system onto a TDL system. On the contrary, it is clear that this is a very unreasonable way of acting in general, as it may cause modification that are not codified.

As a matter of fact, Governatori and Rotolo [21] prevented the consequences of such problematic revisions by assuming that rules can be transformed by substituting them with the empty rule or the void rule, as discussed above. When doing so with an abrogation act included in the law, we need to deal with the representation of the act as a meta-rule, which is not possible in an explicit way in TDL. Thus the representation issues are covered, but the modification needs to be articulated completely, and in an explicit way.

We can suppress the direct consequences in the interval between the expiration date before the revision and the expiration date after the revision, and we do not suppress them afterwards. These differences in the operators map the notions of backward and forward abrogations, for suppression of the norm and not of the consequences in the intervals above defined, whilst if we suppress the norm along with its consequences in the interval we have annulments.

It is rather evident that in this way we have still avoided the temporal reasoning issues, and that we preserved the codified operations. If we assume that the generalisation process has been performed on the same domain of the rules and metarules, we have the following (negative) result.

THEOREM 5.7. TDL systems with rule and meta-rule revision can work with any system of meta-rules defined a priori.

Theorem 5.7 means that when we choose a predefined meta-rule system we can always accommodate it in a TDL system. However to force this to work we need revision. If we assume to codify correctly the behaviour of meta-rules as means to modify the law, we then have this consequence: only codified behaviours actually work, in such a system, as we cannot modify the rules and metarules freely. Is there any possible structural negative effect of this different choice, which could be assumed to be a further reason to choose codified changes? We can prove the following result.

THeorem 5.8. Given a legal system version $L S(i)$, and a legal system version $L S(i+1)$, there exists a set of rules and meta-rules with base revision operators that brings the system from $L S(i)$ to $L S(i+1)$.

Combined with Theorem 5.7, the result of Theorem 5.8 has to be interpreted as follows: the codification of norm changes is necessary for it restricts the ways that are allowed to change the law in a specific legal system.

Conclusively, the choice of extending with the temporary rules the system introduced by Governatori and Rotolo [21] is justified by the need for systems able to remain stable over time, whilst preserving general principles of a legal system and permitting codified changes in the system itself. The hypothesis of accommodating non-codified changes within a system is clearly non-practical. Furthermore, we are strongly interested in understanding the complexity of the reasoning processes in these systems. We should mention that the complexity of general reasoning within the Governatori and Rotolo's framework has not yet been investigated. Theorem 5.4 proves, however, that the decidability condition and the computational complexity of the decision processes for annulment and abrogation operators on temporary laws fully depend on the corresponding conditions on the general operators of annulment and abrogation, due to the linearity of the translation process.

\section{CONCLUSIONS AND FURTHER WORK}

In this paper we extended a model of legal abrogations and annulments in Defeasible Logic adding similar operations acting on temporary norms. A temporary norm is a norm that has a pre-defined expiration time. Above the possibility of changing a norm by annulment or abrogation, it is also possible to modify a temporary norm by anticipating or extending its expiration time. Although it is rather intuitive that an operation of anticipation shares the majority of its properties with abrogation, it is not evident that something similar also happens when we change a norm by moving forward its expiration date. We argue, in particular, that there are two distinct ways of moving backward and two of moving forward the expiration date of a norm, which consist in suppressing the norm (when anticipating) with all the future consequences of the norm locked onward, or retaining the original expiration time for norm lock and the anticipation date for the sole consequences. Moreover, as it happens for instance for tax law, the parliament forces the value limits for the future of a given moment, but when anticipates an expiration date of a temporary norm usually acts on favour of citizens, then preserving the past norm for everyone. On the other way around, if we move the law expiration date forward, we move on the expiration date the consequences but not the norm per se, obtaining different effects when we suppress norm and consequences only at the new expiration date.

We showed that the framework of Governatori and Rotolo [21] accommodates also changes to temporary norms, defined in an 
extension of the framework itself. We also showed that the operations of annulment and abrogation can be used to describe the process of re-positioning the expiration date of temporary norms, but when this is done, the proposed new framework requires a temporal reasoning machinery, that is very undesirable with the defeasible non-monotonic reasoning framework, since simple polynomial machineries can become exponential, and the corresponding computational complexity can devise NP Problems. The process of transformation that is employed to map temporary norm changes onto the Governatori and Rotolo's framework, is linear, and the decision is therefore computable. On top of this, the changing metarules are overwhelmed if we accept changes to the rules based on the extension to annulment and abrogation techniques previously introduced. This is also a validation of the approach of previous work [21] in the specific case of temporary norms.

Many aspects of the changes to temporary norms have not been yet dealt with. On the one hand, the study of temporary norms should also be extended to delegated legislation and to the corresponding issues related to institutional power against that legislation. For instance, when a government decree is issued, in Italy, it expires sixty days after the issuing date. During this period of time, the parliament can convert the decree into a national law, essentially transforming a temporary norm into a persistent one.

An open issue is the comparison to other formal approaches to change, as, e.g., in the studies about revocation of permissions [11]. This investigation has dealt with problems like the persistency of effects of decisions made by persons who left a job after different events, like resignment, retirement, or firing. Different ways of ceasing being part of an organisation can have different effects.

We are taking further this study in two directions. One is the analysis of decidability and complexity issues related to the formalism. In fact, no study has been carried out yet on the decidability of the general framework introduce by Governatori and Rotolo [21], and the computational properties of the extension presented here fully depend on these properties. A general framework based on TDL is under preparation, which aims at accommodating an exhaustive classification of the possible ways in which a normative system can change over time.

\section{ACKNOWLEDGMENTS}

Antonino Rotolo has received funding from the European Union's Horizon 2020 research and innovation programme under the Marie Skodowska-Curie grant agreement No 690974 for the project MIREL: MIning and REasoning with Legal texts.

\section{REFERENCES}

[1] C.E. Alchourrón and E. Bulygin. The expressive conception of norms. In Risto Hilpinen, editor, New Studies in Deontic Logic, pages 95-125. D. Reidel, 1981.

[2] C.E. Alchourrón, P. Gärdenfors, and D. Makinson. On the logic of theory change: Partial meet contraction and revision functions. Journal of Symbolic Logic, 50:510-530, 1985.

[3] C.E. Alchourrón and D.C. Makinson. Hierarchies of regulations and their logic. In Risto Hilpinen, editor, New Studies in Deontic Logic, pages 125-148. D. Reidel, 1981.

[4] C.E. Alchourrón and D.C. Makinson. The logic of theory change: Contraction functions and their associated revision functions. Theoria, 48:14-37, 1982.

[5] G. Antoniou, D. Billington, G. Governatori, and M.J. Maher. Representation results for defeasible logic. ACM Transactions on Computational Logic, 2(2):255287, 2001.

[6] G. Antoniou, D. Billington, G. Governatori, and M.J. Maher. Embedding defeasible logic into logic programming. Theory and Practice of Logic Programming, 6(6):703735, 2006.
[7] M. Araszkiewicz and K. Pleszka, editors. Logic in the Theory and Practice of Lawmaking, volume 2 of Legisprudence Library. Springer, 2015.

[8] N. Bassiliades, G. Antoniou, and I. Vlahavas. A defeasible logic reasoner for the semantic web. International fournal on Semantic Web and Information Systems, 2(1):1-41, 2006.

[9] G. Boella, G. Pigozzi, and L. van der Torre. A normative framework for norm change. In Proceedings of AAMAS 2009, pages 169-176, 2009.

[10] G. Boella, G. Pigozzi, and L. van der Torre. Agm contraction and revision of rules. fournal of Logic, Language and Information, 25(3-4):273-297, 2016.

[11] M. Cramer and G. Casini. Postulates for revocation schemes. In Matteo Maffei and Mark Ryan, editors, Proc ETAPS 2017, pages 232-252. Springer, 2017.

[12] R. de Nevers. Imposing international norms: Great powers and norm enforcement. International Studies Review, 9(1):53-80, 2007.

[13] G. Governatori, J. Hulstijn, R. Riveret, and A. Rotolo. Characterising deadlines in temporal modal defeasible logic. In Proc. Australian AI 2007, pages 486-496, 2007.

[14] G. Governatori, M.J. Maher, D. Billington, and G. Antoniou. Argumentation semantics for defeasible logics. Fournal of Logic and Computation, 14(5):675-702, 2004.

[15] G. Governatori, F. Olivieri, A. Rotolo, S. Scannapieco, and M. Cristani. Picking up the best goal - an analytical study in defeasible logic. In Proc. RuleML 2013, pages $99-113,2013$.

[16] G. Governatori, F. Olivieri, S. Scannapieco, and M. Cristani. Superiority based revision of defeasible theories. In Proc. RuleML 2010, pages 104-118, 2010.

[17] G. Governatori, F. Olivieri, S. Scannapieco, A. Rotolo, and M. Cristani. The rationale behind the concept of goal. Theory and Practice of Logic Programming, 16(3):296-324, 2016.

[18] G. Governatori, M. Palmirani, R. Riveret, A. Rotolo, and G. Sartor. Norm modifications in defeasible logic. In Proc. FURIX 2005, pages 13-22. IOS Press, 2005.

[19] G. Governatori, M. Palmirani, R. Riveret, A. Rotolo, and G. Sartor. Variants of temporal defeasible logic for modelling norm modifications. In Proc. ICAIL 2007, pages 155-159. ACM Press, 2007.

[20] G. Governatori and A. Rotolo. Changing legal systems: Abrogation and annulment. Part I: Revision of defeasible theories. In Proc. DEON 2008, pages 3-18, 2008.

[21] G. Governatori and A. Rotolo. Changing legal systems: Legal abrogations and annulments in defeasible logic. Logic fournal of the IGPL, 18(1):157-194, 2010.

[22] R. Guastini. Teoria e dogmatica delle fonti. Giuffré, Milan, 1998.

[23] T.C. Halliday and B.G. Carruthers. The recursivity of law: Global norm making and national lawmaking in the globalization of corporate insolvency regimes. American fournal of Sociology, 112(4):1135-1202, 2007.

[24] S.O. Hansson. Belief Base Dynamics. Phd thesis, Uppsala University, 1991.

[25] H.L.A. Hart. The Concept of Law. Clarendon, Oxford, 1994

[26] O.A. Hathaway. Path dependence in the law: The course and pattern of legal change in a common law system. Iowa Law Review, 86(2):601-633, 2001.

[27] J. Horty. Norm change in the common law. In S.O. Hansson, editor, David Makinson on Classical Methods for Non-Classical Problems, Outstanding Contributions to Logic. Springer Netherlands, 2013.

[28] HJ. Kelsen. General Theory of Norms. Clarendon, Oxford, 1991.

[29] M.J. Maher. Propositional defeasible logic has linear complexity. Theory and Practice of Logic Programming, 6(1):691-711, 2001.

[30] R.H. Marín and G. Sartor. Time and norms: A formalisation in the event-calculus. In Proc. ICAIL'99, ICAIL '99, pages 90-99, New York, NY, USA, 1999. ACM.

[31] P.E. Navarro and J.L. Rodriguez. Legal Dynamics, pages 196-240. CUP, 2014.

[32] B. Nebel. A knowledge level analysis of belief revision. In Proc. KR'89, pages 301-311. Morgan Kaufmann, 1989.

[33] D. Nute. Defeasible reasoning. In Proceedings of 20th HICSS. IEEE press, 1987.

[34] F. Olivieri, G. Governatori, S. Scannapieco, and M. Cristani. Compliant business process design by declarative specifications. In Proc. PRIMA 2013, pages 213-228, 2013.

[35] A. Rotolo. Retroactive legal changes and revision theory in defeasible logic. In Proceedings of DEON 2010, pages 116-131, 2010

[36] W. Sandholtz. Dynamics of international norm change: Rules against wartime plunder. European fournal of International Relations, 14(1):101-131, 2008.

[37] G. Sartor. Legal Reasoning. Springer, Dordrecht, 2005.

[38] K. Stiles and W. Sandholtz. International Norms and Cycles of Change. 2009.

[39] A. Stolpe. Norm-system revision: theory and application. Artificial Intelligence and Law, 18(3):247-283, 2010.

[40] L. van der Torre, G. Boella, and H. Verhagen, editors. Normative Multi-agent Systems, Special Issue of JAAMAS, vol. 17(1), 2008.

[41] P.J. Wahlbeck. The life of the law: Judicial politics and legal change. Journal of Politics, 59(3):778-802, 1997.

[42] O. Weinberger. The theory of legal dynamics reconsidered. Ratio furis, 4:18-35, 1991.

[43] G.R. Wheeler and M. Alberti. No revision and no contraction. Minds and Machines, 21(3):411-430, 2011. 